\title{
Multibeam Bathymetric Data Quality Control based on Robust Least Square Collocation of Improved Multi-quadric Function
}

\author{
Shengping Wang ${ }^{1,2, *}$, Liwen Dai ${ }^{2}$, Ziyin $\mathrm{Wu}^{1}$, Jiabiao $\mathrm{Li}^{1}$ and Yongqi Wei ${ }^{3}$ \\ ${ }^{1}$ Key Laboratory of Submarine Geosciences, Second Institute of Oceanography, Hangzhou 310012, China \\ ${ }^{2}$ Faculty of Geomatics, East China University of Technology, Nanchang 330013, China \\ ${ }^{3}$ School of Civil and Environmental Engineering, University of California, Los Angeles, California 951593, USA
}

Received 1 February 2018; Accepted 27 July 2018

\begin{abstract}
Abnormal value of the multibeam echosounder system is the main influencing factor of accuracy of hydrographic survey and mapping accuracy of seabed topography. Good functional and stochastic models for multibeam bathymetric data processing are necessary to protect accurate display of multibeam bathymetric data in seabed and safety navigation of ships. This study proposed a robust least square collocation method based on the improved multi-quadric function to represent the variation trend of seabed topography accurately and disclose the real seabed topography along with the systematic and random problems of nonsteady multibeam bathymetric data of seabed topography. Fitting methods of seabed topography trend surface and the elimination of trend items were analyzed. The solving method of the empirical covariance function in the least square collocation and the method to eliminate abnormal values in multibeam bathymetric data were proposed. Validity of the proposed method was proved by an experiment. Results demonstrate that the fitted trend terms in the improved multi-quadric function can reflect the overall trend changes of the study area effectively. The applied robust least square collocation method can effectively reflect the systematicness and randomness of nonsteady bathymetric data of seabed topography. This method can overcome the influences of gross errors or abnormal points on the multibeam bathymetric data and can thus increase the accuracy of the estimation points. Compared with the traditional quadratic polynomial surface fitting method, the proposed method is superior in reflecting the overall trend changes in seabed topography, quality of multibeam bathymetric data, and mapping accuracy of seabed topography. This study can increase the detection capacity and effects of seabed topography at a high working efficiency and lay foundations for scientific research and marine exploration in the future.
\end{abstract}

Keywords: Multi-quadric function, trend term, seabed topography, least square collocation, multibeam bathymetric data

\section{Introduction}

The intervention of modern marine high technology has rapidly developed seabed topographic survey technologies. At present, multibeam echosounder system (MBES) is the mostly used marine mapping equipment. MBES, which is a complicated combined system of multiple sensors, is the high integration of multiple technologies, including modern signal processing, high-resolution display, high-accuracy navigational positioning, and digital sensor technologies [1]. Data collected by MBES form are high-accuracy, highdensity, and full-coverage bathymetric point datasets [2-3]. Multibeam bathymetric survey collects data under a dynamic environment, thereby inevitably resulting in abnormal data due to such factors as instrument noises, marine situations, and ship veering [4-5]. These abnormal data in the survey may further cause false seabed topography, which in turn results in certain difference between the seabed bathymetry and the actual seabed topography.

To control the quality of multibeam bathymetric data and protect the accuracy of seabed bathymetry, data collected by MBES must be preprocessed [6-7]. False signals should be

\footnotetext{
*E-mail address: 37452116@163.com

ISSN: $1791-2377$ @ 2018 Eastern Macedonia and Thrace Institute of Technology. All rights reserved. doi:10.25103/jestr.113.19
}

determined and eliminated, whereas real data should be retained for future data processing study and making necessary preparations of mapping. Considering the systematicness and randomness of nonsteady bathymetric data of seabed topography, multibeam bathymetric data should be processed to establish an improved function model. This step assures that the multibeam bathymetric data can reflect real seabed topography accurately.

Hence, multibeam bathymetric data were preprocessed, analyzed, and tested in this study by a robust least square collocation of improved multi-quadric function. Accordingly, the variation trend of the seabed topography could be represented accurately. The real seabed topography and the systematicness and randomness of nonsteady bathymetric data were reflected. As a result, the drawn seabed bathymetry could provide certain assistance to marine navigation and survey.

\section{State of the Art}

The influencing factors of seabed bathymetry must be analyzed to correct the bathymetric data. Many local and international experts have proposed methods to detect gross errors or abnormal values in multibeam bathymetric data. Methods based on statistical characteristics of neighborhood 
Shengping Wang, Liwen Dai, Ziyin Wu, Jiabiao Li and Yongqi Wei/

\section{Journal of Engineering Science and Technology Review 11 (3) (2018) 139-146}

soundings [8-10], such as those of Ware, Eag, and Cop, can be operated easily. However, parameters in these filtering models must be set artificially, thereby resulting in low degree of automation. Dong et al. [11] proposed a trend surface filtering method based on polynomial surface fitting. However, this method produces incomplete filtering in environments with complicated topographical changes. The method decreases the accuracy of abnormal detection considerably due to its poor resistance to disturbance. Zhang Zhiwei et al. [12] proposed a weight-selection iterative filtering method for trend surface fitting based on robustness. This method can delete jumps and isolated points while retaining seabed microtopography. Thus, the resulting seabed topographic surface is smooth. Nevertheless, this method must consider numerous bathymetric points surrounding the estimation point and cannot reflect the systematicness of nonsteady spatial random field in seabed completely. Rezvani et al. [13] proposed an automatic filtering method based on the robust $M$ estimation. This method compares different equivalent weight functions and gained estimations of each grid point through iterative deduction to obtain the corresponding residual error for recognizing abnormal values. Huang and Vojinovic et al. [14-15] applied the support vector machine into the detection of bathymetric abnormal data. They recognized abnormal values accurately by constructing the continuously changing topographic trend surface, which avoids oblivion of effective seabed topography.

The least square collocation is a method that determines estimation values of the random and non-random parameters in accordance with observation data. This method was applied to study the earth form and gravity field at first [1617]. Subsequently, the method was widely used in many fields, such as geodetic survey and interpolation problem of spatial random field in physical geography [18-19]. XU Wei-Ming et al. [20] proposed to determine non-locating points in hydrographic survey by the least square collocation method and calculate water depth at non-locating points in accordance with the regional depth field information. However, this method cannot detect and eliminate gross errors or abnormal values in multibeam bathymetric data. Chen Zaihui et al. [21] proposed to eliminate gross errors in data by using an adaptive robust least square method. They also pointed out that the accuracy of equivalent weight robust estimation decreases using the least square estimation as the primary value. HD Wang et al. [22] put forward a robust least square collocation method that can delete gross errors or abnormal values in data effectively. However, this method often uses quadratic polynomial for fitting of trend terms, which brings difficulty in characterizing the overall trend changes of seabed topography. Wang L et al. [23] proposed an iterative solving method of robust least square collocation and used the method to estimate parameters of the empirical covariance function. Xie $X$ et al. [24] developed a least square collocation method based on the improved multi-quadric function to estimate the nonsteady spatial random field accurately. This method can reflect the characteristics of the nonsteady spatial random field completely and can thus describe the systematicness of the spatial random field. JT Bjorke [25] calibrated system parameters by using the least square collocation method. This method calculates parameters directly using the least square collocation of the seabed gradient model and can utilize multibeam bathymetric data fully and acquire high parameter estimation accuracy.
The analyses of multibeam bathymetric data processing methods above show that the topographic trend surface constructed through polynomial fitting of trend terms cannot reflect the real seabed topography. A robust least square collocation method based on the improved multi-quadric function was proposed in this study in consideration of the systematicness and randomness of multibeam bathymetric data. In this method, the improved multi-quadric function replaced the traditional quadratic polynomial for fitting of trend terms. With respect to selection of kernel function of the multi-quadric function, the traditional kernel function was developed and the exponential function was used as the improved kernel function. Trend terms were eliminated by the optimal unbiased linear estimation of the improved least square collocation [26-27]. Meanwhile, parameter values of the empirical covariance function and the final results could be estimated accurately. Gross errors or abnormal values in the multibeam data were deleted by the robust least square collocation. Given the least square collocation, the median of bathymetric survey sequence was used as the initial value, and the initial standard error of unit weight was used as the initial variance factor. The covariance and weight arrays of the least square collocation algorithm must be transformed mutually. Thus, the Huber function was applied to calculate the weight factor and update the weight array of the function model. In this way, the equivalent weight would not be 0 . Finally, reliable multibeam bathymetric data were acquired.

The remainder of the study is organized as follows. Section 3 introduces the research route, detailed algorithm contents, and specific solving steps of the proposed method. Section 4 verifies the proposed method by using actual data. A contrastive analysis on three relevant schemes is carried out. Validity and credibility of the proposed method are verified by a series of indexes and actual processing effects. Section 5 elaborates the conclusions. Advantages and characteristics of the proposed method are also introduced.

\section{Methodology}

\subsection{Construction of the primary function}

The basic theory of multi-quadric function model [28-29] is to fit the real data distribution by using any one regular or irregular mathematical surface overlaying and approach at any accuracy. On this basis, the seabed topography was assumed to be a continuously changing curved surface. The function model of the overall seabed topographical trend could be established as follows:

$$
\varsigma(x, y)=\sum_{s=1}^{n} a_{j} Q\left(x, y, x_{j}, y_{j}\right)
$$

Where $n$ is the number of known nodes, $a_{j}$ is the undetermined coefficient, $Q$ is the kernel function, and $\left(x_{j}, y_{j}\right)$ is the node coordinates of the kernel function.

\subsection{Selection of kernel function}

Multibeam stripped bathymetric data have large size and high density of measuring points. When the multi-quadric function was used as the primary function to construct the curved surface, three key problems of kernel function, smoothing factor, and node position must be solved. Among these issues, selection of kernel function can affect the fitting of trend surface considerably. Following the study of 
Du Jiliang [30] in which the improved multi-quadric function was used to describe the GPS deformation field, the present study applied the exponential function as the improved kernel function for curved surface fitting in the multi-quadric function to reflect seabed topography. The kernel function could be expressed as follows:

$$
Q\left(x, y, x_{j}, y_{j}\right)=e^{-\left(k L^{2}\left(x, y, x_{j}, y_{j}\right)\right)^{i}}
$$

Where $i=1.0$ and $k=0.0035-0.0080$. This model developed the kernel function of the classical multi-quadric function. The improved kernel function had strong robustness, which enabled it to fit the seabed curved surface in irregular variation effectively and increase the least square collocation accuracy. Therefore, the variation trend of seabed topography was reflected accurately.

\subsection{Construction of the least square collocation of the improved multi-quadric function}

Equation (1) was changed into the following matrix form:

$$
\varsigma(\lambda, f)=Q a
$$

The multi-quadric function was applied to fit the overall seabed topographical trend. Fitted results were input into the least square collocation. Accordingly, the function configuration model could be gained as follows:

$$
Z=Q a+B x+\Delta
$$

Where $Q a$ is the continuous changing part of the seabed topography, $m$ is the number of nodes, and $Q$ is the kernel function matrix of $m \times n . a$ is the undetermined coefficient vector of $n \times 1, B x$ is the irregular changing part of the seabed topography, and $\Delta$ is the error vector of $m \times 1$.

The optimal unbiased linear solutions to $a$ and $x$ in Equation (4) were calculated as follows:

$$
\left\{\begin{array}{l}
\hat{a}=\left\{Q^{T}\left(B D_{x} B^{T}+D_{\Delta}\right)^{-1} Q\right\}^{-1} Q^{T}\left(B D_{X} B^{T}+D_{\Delta}\right)^{-1}\left(Z-B Z_{X}\right) \\
\hat{x}=Z_{x}+D_{x} B^{T}\left(B D_{x} B^{T}+D_{\Delta}\right)^{-1}\left(Z-Q \hat{a}-B Z_{x}\right) \\
\hat{x}^{\prime}=Z_{x^{\prime}}+D_{x^{\prime} x} B^{T}\left(B D_{x} B^{T}+D_{\Delta}\right)^{-1}\left(Z-Q \hat{a}-B Z_{x}\right)
\end{array}\right.
$$

where $Z_{x}$ is the signal median, $D_{x}$ is the covariance matrix between signals of different measured points, $D_{x^{\prime} x}$ is the covariance matrix between signals in the estimation and measured points, and $\hat{x}^{\prime}$ is the estimating signal value. $D_{x}$ and $D_{x^{\prime} x}$ were given by the empirical covariance function. The Gaussian function is generally applied for fitting [31]. The Gaussian model was expressed as follows:

$$
F(d)=F(0) e^{-U^{2} d^{2}}
$$

Where $F(0)$ and $U$ are undetermined coefficients, $d$ is the distance between two points, and $U$ is the spatial correlation scale. Supposing functions were isotropic and were only related with distance. $F(0), F(d)$, and $U$ were calculated by:

$$
\left\{\begin{array}{l}
F(0)=\left(\sum_{i=1}^{N} r_{i}^{2}\right) / n \\
F(d)=\left[r_{i} \times r_{j}\right] / n_{d}
\end{array}\right.
$$

where $r=Z-Q a$ and $n$ is the total number of measured points. $n_{d}$ is the total number of point pairs with a distance of $d$.

\subsection{Solving the robust least square collocation based on} the improved multi-quadric function

\subsubsection{Initial value calculation of the median parameter method}

The median parameter median $\left(\hat{a}^{j}\right)$ in Equation (1) was solved. On this basis, the median vector $a_{\text {med }}$ of model parameters was solved as follows:

$$
a_{\text {med }}=\left[\operatorname{median}\left(\hat{a}^{1}\right), \text { median }\left(\hat{a}^{2}\right), \operatorname{Lmedian}\left(\hat{a}^{n}\right)\right]
$$

The difference between different solutions and $a_{\text {med }}$ was calculated, $j=1 \mathrm{~L} p$. For the $p$ group of solutions, the following condition was obtained:

$$
\left\{\begin{array}{l}
d a_{j}=a_{j}-a_{\text {med }} \\
\min _{-} d a=\min \left\{\left\|d a_{1}\right\|,\left\|d a_{2}\right\| \mathrm{L}\|d a p\|\right\}
\end{array}\right.
$$

where $\left\|d a_{j}\right\|$ is the quadratic norm of $d a_{j}$. The model parameter vector corresponding to the minimum value min. $d a$ was the solution of median parameter method in this study. The residual correction corresponding to the whole observation vector was calculated, and the residual error was used as the initial value for robust estimation. The initial standard error of unit weight $\left(\sigma_{0}\right)$ was expressed as follows:

$$
\left\{\begin{array}{l}
v_{\text {med }}=\text { median }\left\{\left|v_{1}\right|,\left|v_{2}\right|, \mathrm{L}\left|v_{m}\right|\right\} \\
\sigma_{0}=v_{\text {med }} / 0.6745
\end{array}\right.
$$

\subsubsection{Solving the initial weight matrix of observed values} Multibeam Echosounder System is the main device of bathymetry at present. Bathymetric data have high accuracy and resolution and can meet the requirements of large-scaled digital seabed topographical modeling. MBES easily results in abnormal values of multibeam data due to the complicated situations and instable factors of bathymetry. To acquire high-quality multibeam bathymetric data, gross errors or abnormal values should be detected and deleted. To address this problem, the robust least square collocation was proposed to process multibeam data with gross errors or abnormal values. This method initialized the observation covariance matrix by the median of observed values. It determined estimated parameters, and the median method determined the model observed quantity and mean square error of elements in the matrix. The initial steps of specific variance were as follows:

1. Use the median of bathymetric depth as the initial value of the variance matrix 
Shengping Wang, Liwen Dai, Ziyin Wu, Jiabiao Li and Yongqi Wei /

\section{Journal of Engineering Science and Technology Review 11 (3) (2018) 139-146}

$$
\tilde{Z}=\operatorname{med}\left\{\left|Z_{I}\right|\right\}
$$

2. Calculate the initial residual error of Equation (11)

$$
\Delta Z=Z-\tilde{Z}
$$

The initial variance factor was

$$
\tau_{\Delta Z}=\text { median }(|\Delta Z|) / 0.6745
$$

3. The covariance and weight matrixes in the least square collocation algorithm must be transformed mutually. Thus, the Huber function was used to calculate the weight factor for ensuring that the equivalent weight would not be 0 .

$$
\bar{p}= \begin{cases}1 & \left|\Delta_{Z} / \tau_{\Delta Z}\right| \leq c \\ c /\left|\Delta_{Z} / \tau_{\Delta Z}\right| & \left|\Delta_{Z} / \tau_{\Delta Z}\right|>c\end{cases}
$$

where $c$ is generally $1.5-2.5$.

3.4.3 Model parameter solving of the covariance function Accuracy of empirical covariance function fitting is extremely important to obtain accurate least square collocation results. Two parameter solutions $F(0)$ and $u$ in Equation (7) were solved using the equations above. The updated observation variance matrix was taken into in Equation (8) to obtain the $F(0)$ solution after robustness as follows:

$$
F(0)=\frac{{ }_{i}^{T} p_{0} r}{n}
$$

where $r=Z-Q a$ and $n$ is the total number of measured points. $n_{d}$ is the total number of point pairs with a distance of $d$.

Supposing $s+1$ equal distances existed: $d_{h}=h \times H$, where $H=0,1,2, \mathrm{~L}, s$ and $h$ is the distance of different sections. To offset the influences of gross errors or abnormal data on solving $U$, the updated observation variance matrix was taken into Equation (8). $F\left(u_{h}\right)$ could be calculated as follows:

$$
F\left(d_{h}\right)=\frac{\left[p_{0 i j} \times r_{i} \times r_{j}\right]}{s_{h}}
$$

By introducing the results of Equations (13) and (14) into Equation (7), $U$ could be gained by

$$
U_{i}=\sqrt{\frac{1}{d_{i}^{2}}\left(\ln F(0)-\operatorname{LnF}\left(u_{h i}\right)\right)}
$$

The specific calculation steps of the improved method were as follows:

(1) Initialize the covariance function. The initial values $F(0)=1$ and $U^{(0)}=0$ of the covariance function were given. The median of observation value was used as the initial observation variance, and the trend term was fitted by the multi-quadric function. The results were taken into Equation (5) to obtain estimations $a_{i}$ of the undetermined coefficient.

(2) Trend term in the observation data $Z$ was eliminated using Equation (5), and the signal $x_{i}$ was acquired. The observation variance matrix was updated by the Huber function, followed by statistics on signal covariance. The parameters $F(0)_{i+1}$ and $U_{i+1}$ of model covariance function were gained by solving the covariance function in Section 3.2. The covariance matrix $D_{x}$ was updated.

(3) The updated covariance matrix $D_{x}$ was taken into Equation (6), and the new determined coefficient $a_{i+1}$ was obtained. Therefore, the new least square collocation solution was obtained.

(4) A cyclic computation of this algorithm was given in first three steps. Repeat the steps above until meeting the iterative condition, End the computation condition:

$\left\|F(0)^{(i+1)}-F(0)^{(i)}\right\|<\varepsilon \& \&\left\|U^{(i+1)}-U^{(i)}\right\|<\xi$.

(5) In accordance with the undetermined coefficient $a_{i+1}$, the signal value $\hat{x}^{\prime}$ of the estimation point was acquired from Equation (5). Finally, the estimated depth of water at the measuring points was gained by $\hat{Z}^{\prime}=\hat{x}^{\prime}+Q a_{i+1}$.

\section{Result Analysis and Discussion}

Experimental data were collected from 1,045 bathymetry points in an offshore beach. The curved surface of the study area was constructed using the improved multi-quadric function as the primary function. Among all the points, 32 multibeam bathymetric data points were chosen randomly as the checkpoints. The data distribution is shown in Fig. 1 (the red stars are the checkpoints, and the black points are the measured points). The bathymetric contour map of the study area is shown in Fig. 2.

To verify the validity of the proposed method, the least square collocation method based on quadratic polynomial (method 1), the robust least square collocation method based on multi-quadric function (method 2), and the proposed method were compared. Feasibility and advantages of the proposed method were verified by the residual errors and external character accuracies at the checkpoints. The three methods were used to process multibeam bathymetric data. The residual errors and external character accuracies of the three methods at the checkpoints are listed in Table 1 . The bathymeric residual errors of the three methods at the checkpoints are shown in Fig. 3.

Table 1 shows that the proposed method (the robust least square collocation based on the improved multi-quadric function) was superior in terms of all the indexes. The external character accuracies of the three methods were $1.3814,0.8515$, and $0.8377 \mathrm{~m}$, respectively. Compared with the traditional quadratic polynomial method, the proposed method increased the external character accuracy by 0.5437 $\mathrm{m}$. This finding implied that fitting of trend term based on the multi-quadric function could reflect the overall trend changes of the study area, and stable randomly changing part could be gained after deletion of the trend term. Consequently, accurate results were obtained. 


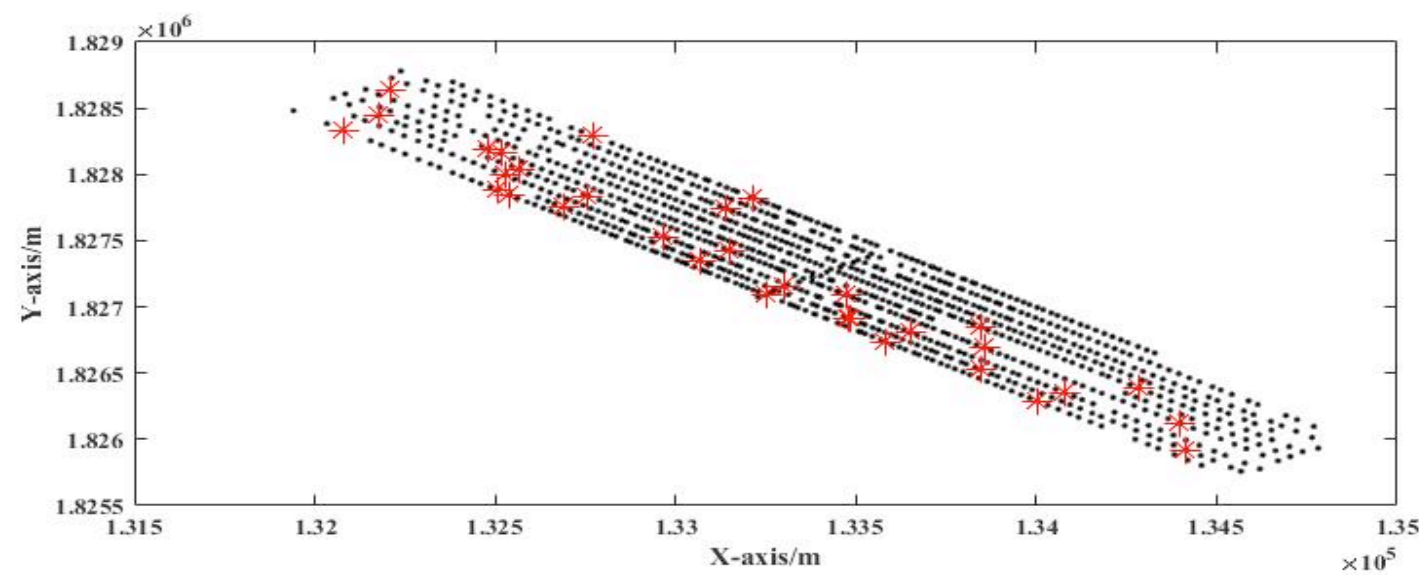

Fig. 1. Data distribution map of multibeam bathymetry

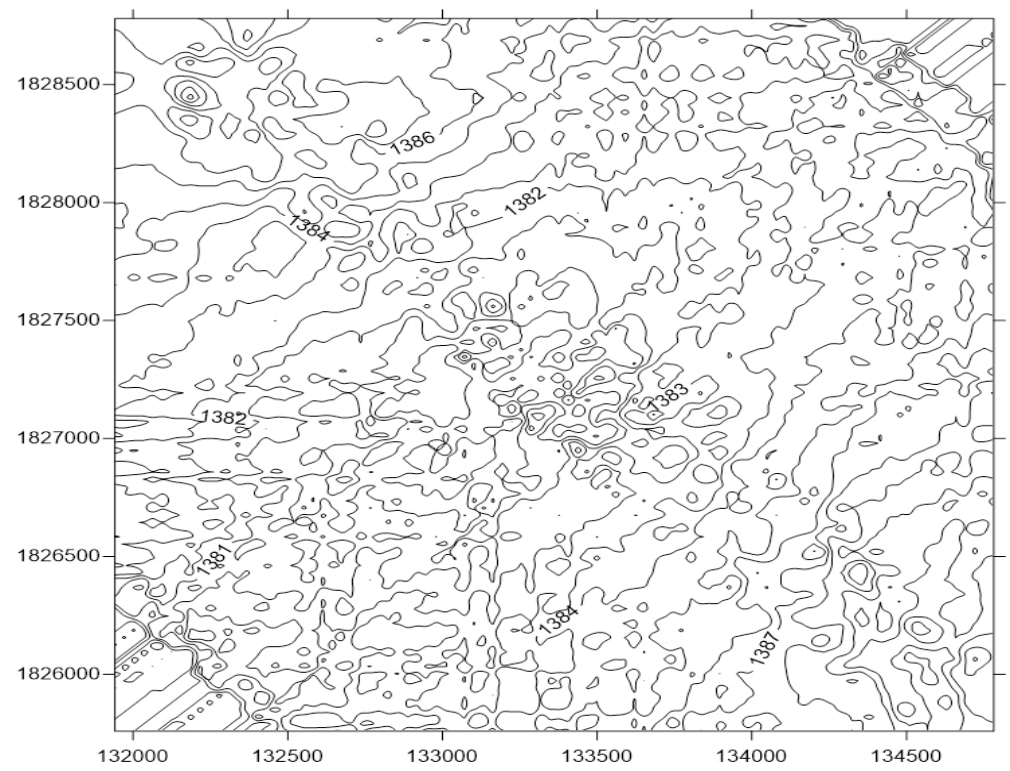

Fig. 2. Bathymetric contour map of the original data

Table 1. Statistics of the residual errors and external character accuracies of three methods at the checkpoints

\begin{tabular}{l|l|l|l}
\hline Methods & Maximum /m & Minimum/m & External character accuracy \\
\hline Method 1 & 3.2275 & -3.5074 & 1.3814 \\
Method 2 & 2.1067 & -2.0860 & 0.8515 \\
The proposed method & 1.9830 & -1.3572 & 0.8377 \\
\hline
\end{tabular}

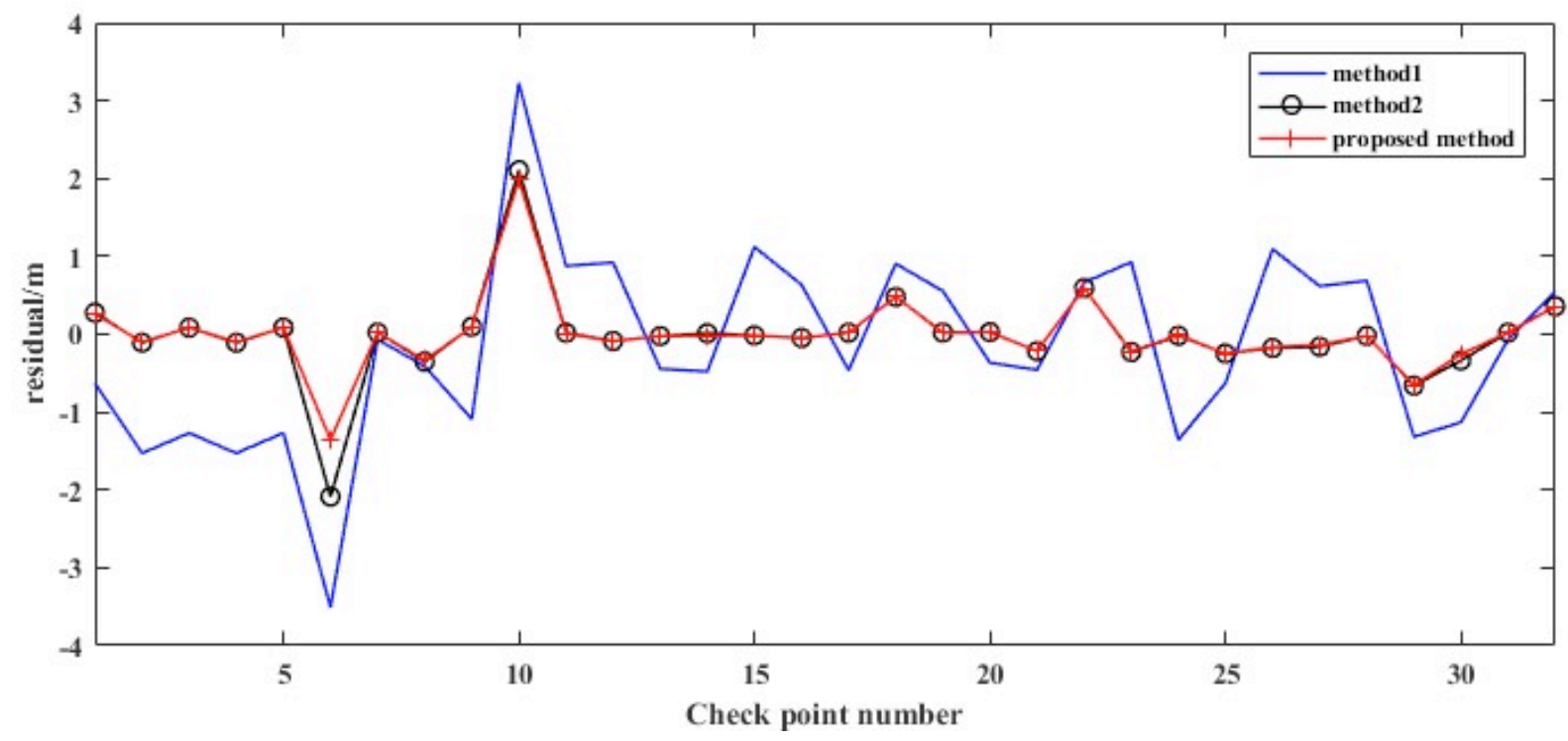

Fig. 3. Comparison of the residual errors of three methods at the checkpoints 
Fig. 3 shows that the residual error of the proposed method was smaller than those of the two other methods, which further verified the validity of trend term fitting based on the improved multi-quadric function. The residual norms of the three methods were calculated as $6.8305,3.2447$, and 2.7223, which further demonstrated that method 1 (the least square collocation based on quadratic polynomial) was the poorest, and method 3 was the best with respect to the estimation effect.

Each point in a triangular irregular network (TIN) represents the original data. The TIN can avoid data redundancy in the environment of flat terrain. Each point in the TIN can express digital elevation characteristics in accordance with topographic feature points. Therefore, it can reflect the continuous covering surface effectively. The Delaunay TIN is conducive to data updating and topographical analysis [32]. Therefore, the Delaunay TINs were generated in this study by using unprocessed multibeam bathymetric data, and the multibeam bathymetric data were processed by the proposed method (Figs. 4 and 5). In the experiment, the bathymetric data corresponding to the equivalent weight $\bar{p} \leq 0.65$ were calibrated as abnormal points.

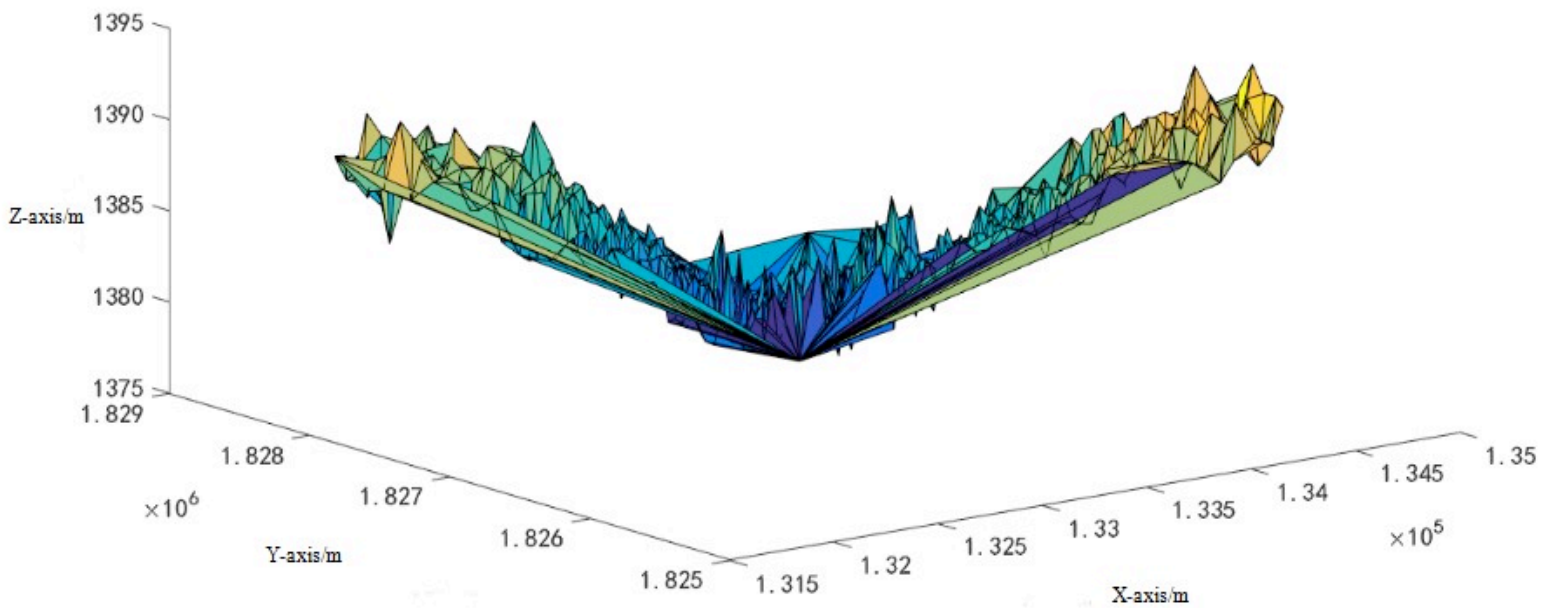

Fig. 4. Diagram of the unprocessed TIN

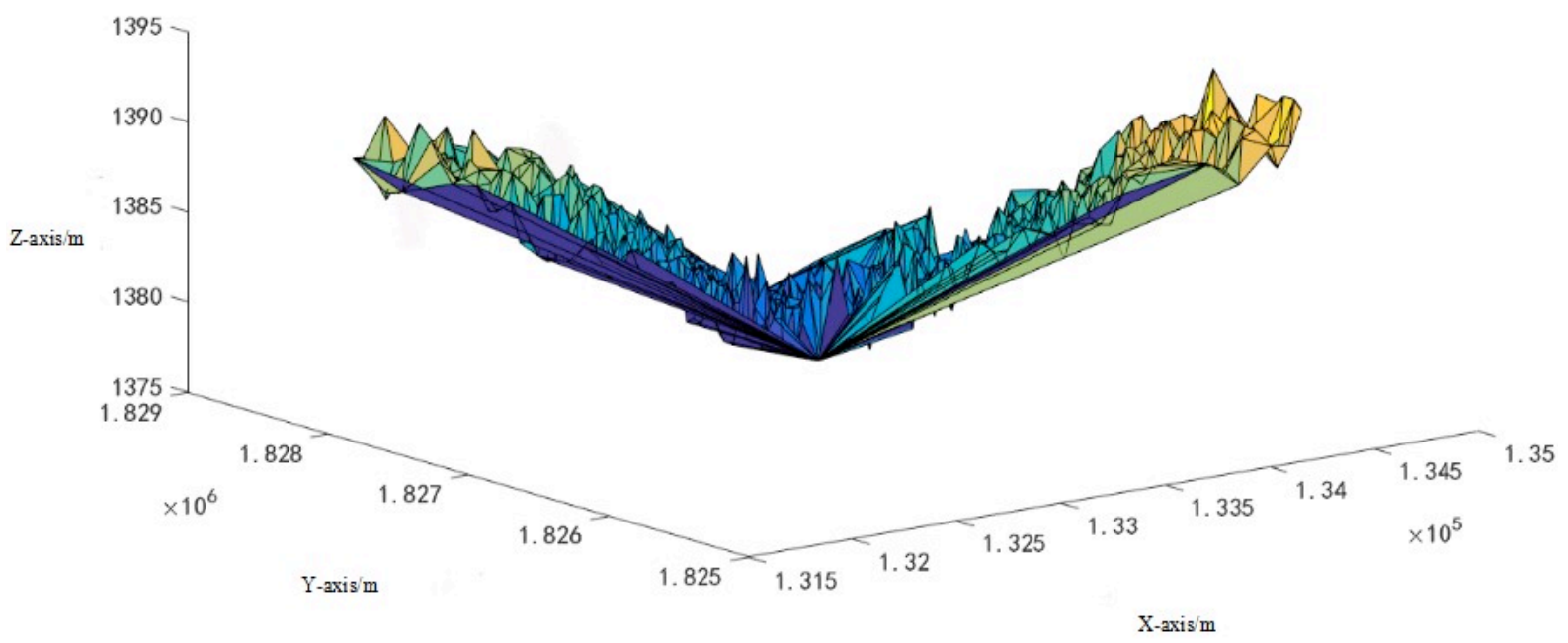

Fig. 5. TIN diagram generated by the proposed method

Figs. 4 and 5 show that the TIN diagram generated by processed data reduced crossing triangles, and the triangles were close to the regular ones. This condition increased the probability to form triangles by the closest points and deleted abnormal data points effectively. The generated TIN diagram could characterize topography accurately.
High-quality DEM can be obtained from TIN interpolation of regular grids. In the current study, DEM diagrams were generated by the original multibeam bathymetric data and the TIN interpolation processed by the proposed method (Figs. 6 and 7). 


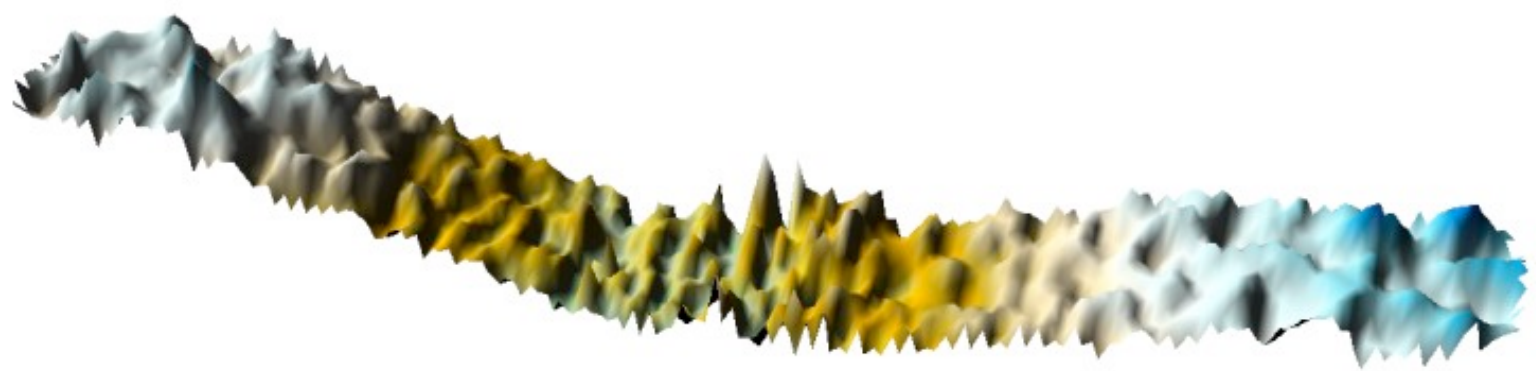

Fig. 6. Unprocessed DEM diagram

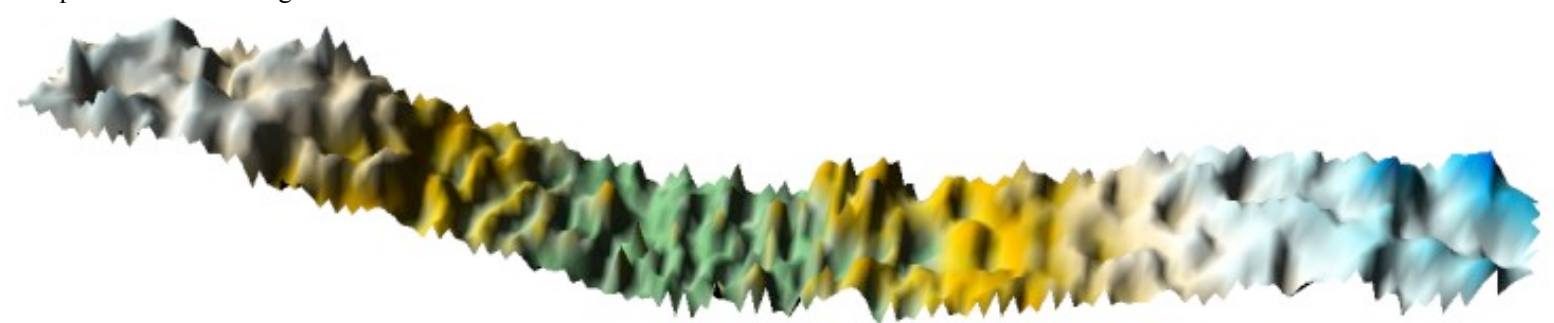

Fig.7. DEM diagram generated after processing

Comparison of Figs. 6 and 7 showed some prominent abnormal values in Fig. 6 accompanied with convex wrinkles at the edges. However, no abnormal convexes were found in Fig. 7 because the edges tended to be smooth. Nevertheless, the change features of seabed microtopography in the study area were retained. Therefore, the constructed seabed topographical model could characterize the real seabed topography accurately.

\section{Conclusion}

This study proposed a robust least square collocation method based on the improved multi-quadric function. This method could protect the quality of multibeam bathymetric data, solve problems in bathymetric data, and represent the real seabed topography. The specific solving algorithm was introduced, and its validity was verified by an experiment. The main conclusions were as follows:

(1) The improved multi-quadric function can represent the overall trend changes of seabed topography accurately. The random part after deletion of the trend term becomes smoother and the acquired signal value is more accurate than before, which further increases the estimation accuracy of the least square collocation.

(2) Abnormal data in multibeam bathymetry influence the real bathymetry. The proposed method can delete gross errors or abnormal data effectively. Thus, this method can protect the quality of multibeam bathymetric data, increase the accuracy of estimation, and reflect the real seabed topology accurately.
(3) High-quality DEM is acquired by transforming the TIN linear interpolation into regular grids.

In this study, the improved method for multibeam bathymetric data processing is proposed by combining theoretical methods and real data. This method improves the quality of bathymetric data and increases the mapping accuracy of seabed topography. It offers reliable bathymetric data and accurate topographic information for marine scientific studies concerning sea charting and coastal engineering in the future. Given the present data size limitation, further studies are still needed to regions with larger data size and more complicated seabed topography than the current ones to develop a highly accurate, highly efficient, and universal function model for multibeam bathymetric data processing.

\section{Acknowledge}

This work was supported by the Open Fund Project of Jiangxi Provincial Digital Land and Environment Laboratory (DLLJ201719), the Fund Project of Jiangxi Provincial Education Department (GJJ170447), the Natural Science Foundation of Jiangxi Province in China (20161BAB 206163), the National Natural Science Foundation of China (41206078), and the Director Fund of Second Institute of Oceanography (JG-1508).

This is an Open Access article distributed under the terms of the Creative Commons Attribution License

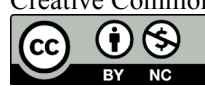

\section{References}

1. Zhou Tian. "Application Approach for CCISD". Master thesis of Harbin Engineering University, China, 2005, pp.5-10.

2. Cutter, George R, L. Berger, and D. A. Demer. "A comparison of bathymetry mapped with the Simrad ME70 multibeam echosounder operated in bathymetric and fisheries modes". Ices Journal of Marine Science, 67(6), 2010, pp.1301-1309.

3. Fezzani, Ridha, et al. "Fusion of Swath Bathymetric Data: Application to AUV Rapid Environment Assessment". IEEE Journal of Oceanic Engineering, PP(99), 2018, pp.1-10.
4. An, L, et al. "Bed Topography of Store Glacier and Fjord, Greenland from High-Resolution Gravity Data and Multi-Beam Echo Sounding". In: $A G U$ Fall Meeting $A G U$ Fall Meeting Abstracts, San Francisco, USA: AGU, 2014, pp.683-688.

5. Rezvani, Mohammad Hadi, A. Sabbagh, and A. A. Ardalan. "Robust Automatic Reduction of Multibeam Bathymetric Data Based on Mestimators". Marine Geodesy, 38(4), 2015, pp.327-344. 
Shengping Wang, Liwen Dai, Ziyin Wu, Jiabiao Li and Yongqi Wei /

\section{Journal of Engineering Science and Technology Review 11 (3) (2018) 139-146}

6. Barreyre, Thibaut, et al. "Temporal variability and tidal modulation of hydrothermal exit-fluid temperatures at the Lucky Strike deepsea vent field, Mid-Atlantic Ridge". Journal of Geophysical Research Solid Earth, 119(4), 2014, pp.2543-2566.

7. Fabio Sacchetti, et al. "Geomorphology of the Irish Rockall Trough, North Atlantic Ocean, mapped from multibeam bathymetric and backscatter Data". Journal of Maps, 7(1), 2011, pp.60-81.

8. Du, Z., Wells, D., Mayer, L., "An approach to automatic detection of outliers in multibeam echo sounding data". Hydrographic Journal, (79), 1996, pp.19-23.

9. Perry, R. "Scientific and Hydrographic use of the Bathymetric SWATH Survey System". In: OCEANS 82, Washington, USA: Oceans, 1982, pp.396-401.

10. Ware, Colin, W. Knight, and D. Wells. "Memory intensive statistical algorithms for multibeam bathymetric data". Computers \& Geosciences, 17(7), 1991, pp.985-993.

11. Dong, J., Ren, L., S., "Filter of MBS Sounding Data Based on Trend Surface". Hydrographic Surveying and Charting, China, 27(6), 2007, pp. 25-28.

12. Zhang, Zhiwei, et al. "Application of robust estimation for detecting outliers of multibeam data". Journal of Liaoning Technical University, 35(07), 2016, pp.755-758.

13. Rezvani, M. H., Sabbagh, A., Ardalan, A. A., "Robust Automatic Reduction of Multibeam Bathymetric Data Based on Mestimators". Marine Geodesy, 38(4), 2015, pp.327-344.

14. Huang, X., et al. "Application of least square support vector machine to detecting outliers of Multi-beam data". Geomatics \& Information Science of Wuhan University, 35(10), 2010, pp.11881187.

15. Vojinovic, Zoran, et al. "A machine learning approach for estimation of shallow water depths from optical satellite images and sonar measurements". Journal of Hydroinformatics, 15(4), 2013, pp.1408-1424.

16. Jarmołowski, Wojciech. "Least squares collocation with uncorrelated heterogeneous noise estimated by restricted maximum likelihood". Journal of Geodesy, 89(6), 2015, pp.577-589.

17. Yang, Y., A. Zeng, and J. Zhang. "Adaptive collocation with application in height system transformation". Journal of Geodesy, 83(5), 2009, pp.403-410.

18. Noroozi, Ali, and M. A. Sebt. "Spherical interpolation method of emitter localisation using weighted least squares". Iet Signal Processing, 10(8), 2016, pp.841-854

19. Krypiakgregorczyk, Anna, P. Wielgosz, and W. Jarmołowski. “A new TEC interpolation method based on the least squares collocation for high accuracy regional ionospheric maps". Measurement Science \& Technology,28(4), 2017, pp. 045801
20. Wei-Ming, X. U., and Y. C. Liu. "Methods of Determining NonPosition Points in Hydrographic Survey". Journal of System Simulation, 19(20), 2007, pp.4612-4615.

21. Chen, Zai Hui, and L. U. Xiao-Feng. "Gross Error Elimination in DEM Data Based on Adaptive Robust Least Squares Estimation". Hydrographic Surveying and Charting, China, 26(6), 2006, pp.1517.

22. Wang, H. D., H. Z. Chai, and D. M. Zhao. "Research on seabed terrain generation based on robust least-squares collocation". Journal of System Simulation, 22(9), 2010, pp.20912080 .

23. Wang, Leyang, and H. Chen. "Multi-beam Bathymetry Data Processing Using Iterative Algorithm of Robust Least Squares Collocation". Acta Geodaetica Et Cartographica Sinica,46(5), 2017, pp.658-665.

24. Xie, X., et al. "A Refined Least Squares Collocation Method Based on Multiquadric Function”. Wuhan Daxue Xuebao,43(4), 2018, pp.592-598.

25. Bjorke, J. T. "Computation of calibration parameters for multibeam echo sounders using the least squares method". IEEE Journal of Oceanic Engineering, 30(4), 2005, pp.818-831.

26. Sozzi, R, et al. "Quality control and gap-filling of PM10 daily mean concentrations with the best linear unbiased estimator". Environmental Monitoring \& Assessment, 189(11), 2017, pp.562.

27. Haq, Abdul, et al. "Best linear unbiased and invariant estimation in location-scale families based on double-ranked set sampling". Communications in Statistics, 45(1), 2016, pp.25-48.

28. Hardy, Rolland L. "Multiquadric equations of topography and other irregular surfaces". Journal of Geophysical Research, 76(8), 1971, pp.1905-1915.

29. Panda, Bhanumati, S. Kumar, and R. K. Misra. "Solving Singularly Perturbed Problems Using Multi-quadric/Inverse Multi-quadric Radial Basis Function Method", Indian Journal of Industrial and Applied Mathematics, 7(1), 2016, pp.43-57.

30. Du Jiliang. "Application Approach for CCISD". Master thesis of Earthquake Prediction Institute of China Earthquake Administration, China, 2016, pp.8-10.

31. Jiang, Zai Sen, and J. N. Liu. "The Method for Establishing the Strain Field and Velocity Field of Crustal Movement Using Least Squares Collocation". Chinese Journal of Geophysics, 53(3), 2010 , pp.380-389.

32. Tucker, Gregory E., et al. "An object-oriented framework for distributed hydrologic and geomorphic modeling using triangulated irregular networks". Computers \& Geosciences, 27(8), 2001,pp.959-973. 